\title{
Einfluß der Dicke von Borosilikatglas als Substratgegenkörper auf den mechanischen Spannungszustand von piezoresistiven Silizium- Hochdruck-Messelementen
}

\author{
Patrick Heinickel, Roland Werthschützky \\ Institut für Elektromechanische Konstruktionen, Technische Universität Darmstadt \\ Merckstraße 25, 64283 Darmstadt, Deutschland \\ Kontakt P. Heinickel: Tel: 0615116 3795, E-Mail: p.heinickel@emk.tu-darmstadt.de
}

\section{Kurzfassung}

Das Verbundelement als piezoresistiver Silizium Drucksensors für den Hochdruckbereich bis 5.000 bar (500 MPa) basiert auf einem neuartigen Prinzip der mechanischen Verspannung infolge der allseitigen Druckbelastung des prozessierten Silizium-Chips mit einem mechanisch fehlangepassten Glasgegenkörper. Der analytische Ansatz zur Beschreibung des Verbundelements mit Ableitung des Einflusses des Dickenverhältnisses auf die Empfindlichkeit wird in dieser Veröffentlichung gezeigt. Darauf wird der theoretische Ansatz durch messtechnische Charakterisierung von sieben Verbundelementen mit drei unterschiedlichen Dickenverhältnissen bestätigt. Es zeigt sich ein nichtlineares Verhalten bei den Elementen mit den dicksten Glasgegenkörpern, die mehr als doppelt so dick wie das Silizium sind.

\section{Motivation}

Aktuell nimmt die Anzahl der industriellen Anwendungen im Hochdruckbereich von größer $10 \mathrm{MPa}$ (100 bar bzw. ca. 1.450 psi) ständig zu. Bei Massenanwendungen, wie z.B. in der Automobilindustrie, wird beim Bremssystem und Assistenzsystemen mit Druck bis $35 \mathrm{MPa}$ gearbeitet. Bei der Common-RailEinspritzung beträgt der Rail-Druck derzeit etwa $160 \mathrm{MPa}$ auf der Straße und zwischen $200 \mathrm{MPa}$ und $300 \mathrm{MPa}$ in der Entwicklung Im Maschinenbau, aber auch in der Lebensmittel- und Verfahrenstechnik (Petrochemie) werden Prozesse mit einem Druck von $500 \mathrm{MPa}$ bzw. sogar von $1.500 \mathrm{MPa}$ und mehr etabliert.

Hochdruck-Sensoren nach dem Stand der Technik bestehen meistens aus Metall- oder KeramikVerformungskörpern, die mit Dehnungssensoren bestückt sind. Auf der einen Seite handelt es sich um aufgeklebte metallische Folien-Dehnungsmesssteifen (DMS), auf der anderen Seite wird die Dünnfilmtechnik, auf dem Verformungskörper abgeschiedene metallische Widerstandsschichten als Funktionsgebiete, angewendet. Bei diesen Hochdrucksensoren umfasst der Überlastbereich im Normalfall maximal $120 \%$ des Nenndruckbereichs. Bei Überlastungen oberhalb des ausgewiesenen Bereichs kommt es zur plastischen Verformung des Metallverformungskörpers und somit zu einem nicht reparablen Defekt des Sensors. Aufgrund der zugunsten der Stabilität und Linearität monolithisch hergestellten Verformungskörper, sind diese Sensoren darüber hinaus sehr teuer.

Die meistverbreitete Technik zur Messung von Druck im Normaldruckbereich sind piezoresistive SiliziumDrucksensoren. Aufgrund des verwendeten monokristallinen Siliziums als Funktionswerkstoff ergeben sich eine Reihe von Vorteilen wie günstige Massenproduktion, kleine Baugrößen und gute Miniaturisierbarkeit, einfache Formgebung, Langzeitstabilität und vergleichbar hohe Empfindlichkeit. Aufgrund der Verwendung einer im Silizium gefertigten Druckplatte, die je nach Druckbereich nur wenige $\mu \mathrm{m}$ dick ist, ergibt sich für diese Silizium-Drucksensoren ein Einsatzdruckbereich von $1 \mathrm{kPa}$ bis maximal $100 \mathrm{MPa}$. 


\section{Funktion und Aufbau des neuartigen Hochdruck-Messelementes}

Die Vorteile von Silizium als Funktionswerkstoff sollen zukünftig auch im Hochdruckbereich genutzt werden können. Daher wurde ein piezoresistives Hochdruck-Messelement für einen Nenndruck bis $500 \mathrm{MPa}$ (5.000 bar) entwickelt [1, 2, 3]. Das neuartige Hochdruck-Messelement, Verbundelement genannt, besteht aus einem prozessierten Siliziumchip ohne Rückseitenkavität, das anodisch an einen Gegenkörper gebondet ist (Bild 1). Um mechanische Stabilität und Überlastfestigkeit in höchsten Druckbereichen zu gewährleisten, wird das Verbundelement allseitig mit Druck belastet. Dies führt dazu, dass im Verbundelement keine für das Material und die Verbindung kritischen Zugspannungen auftreten, sondern lediglich Kompressionen. Aufgrund des mechanisch fehlangepassten Gegenkörpers komprimiert sich dieser im Vergleich zum Silizium weichere Körper stärker. Wegen einer stetig verlaufenden mechanischen Dehnung an der Grenzschicht verbiegt sich das Verbundelement bei hydrostatischer Belastung schalenförmig. Dieser Zustand der mechanischen Verspannung des Verbundelementes wird mittels im Silizium implantierter piezoresistiver Widerstände zur Messung des Drucks angewendet. Als WHEATSTONE'sche Vollbrücke sind diese vier im Silizium implantierten piezoresistiven Widerstände verschaltet, um eine möglichst stabile elektrische Messung durchführen zu können.

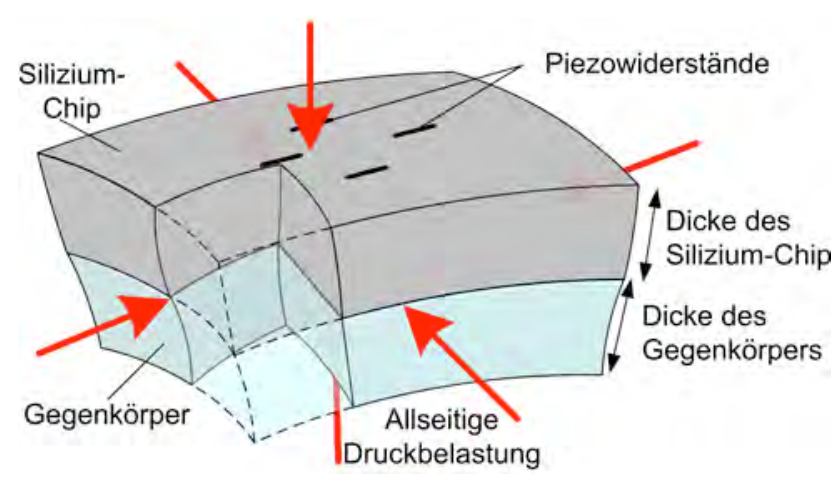

Bild 1: Schalenförmig verbogenes Verbundelement als neuartiges HochdruckMesselement aufgrund hydrostatischer Druckbelastung mit einer Kantenlänge von ca.

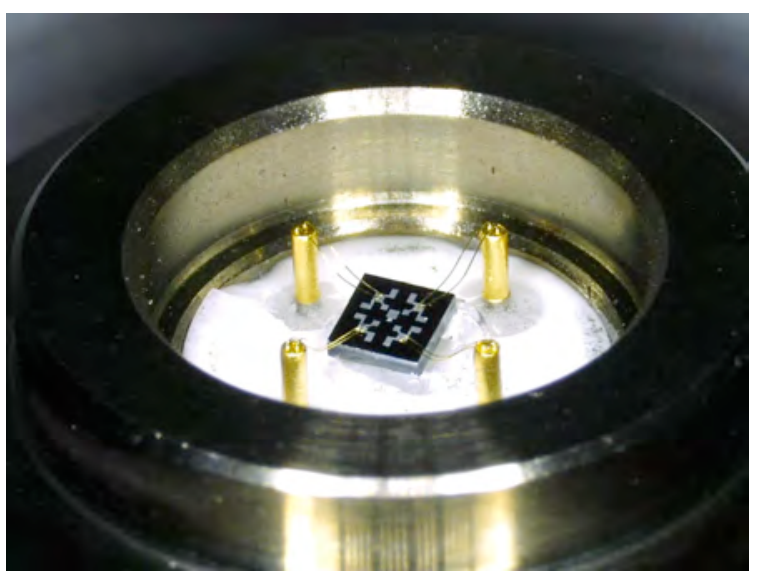

Bild 2: Foto eines Musteraufbaus: Verbundelement aufgeklebt auf einer Glas-KeramikDurchführung und elektrisch kontaktiert mit Gold-Bonddraht

Da sich das Verbundelement im Bereich des zu messenden Druckmediums befindet, müssen elektrisch isolierend und gleichzeitig gegen Atmosphärendruck dichtend, vier elektrische Verbindungen von der Hochdruck- zur Umgebungsdruck-Seite bereitgestellt werden. Diese Funktion übernehmen im Normaldruckbereich bis ca. 100 bar Glasdurchführungen nach Stand der Technik. Für den in dieser Arbeit geforderten erweiterten Druckbereich bis 5.000 bar, sind speziell angepasste Varianten von elektrischen Durchführungen erforderlich. Bild 2 zeigt einen Musteraufbau mit kontaktiertem Verbundelement und einer weiterentwickelten Variante einer elektrischen Glas-Keramik-Durchführung [12]. Die durch verbesserte Materialkombinationen stark gesteigerte mechanische Stabilität zeigt bei durchgeführten Druckbelastungen eine Dichtigkeit und Funktion bis zum maximal in dieser Einrichtung erzeugbaren Druck von 5.000 bar.

\section{$3 \quad$ Analytische Beschreibung des neuartigen Messeffektes}

\subsection{Lösungsansatz}

Um die Optimierungen des Verbundelementes als Hochdruck-Messelement systematisch entwickeln zu können, wird ein Ansatz zur analytischen Beschreibung der mechanischen Verspannung aufgestellt [2, 3]. Da das Verbundelement allseitig mit Druck belastet wird, wird ein mehrdimensionaler Ansatz auf Basis eines Ansatzes zur Erhaltung der mechanischen Energie in einem abgeschlossenen System gewählt. Die Systemgrenze ist in diesem Fall die Außenfläche des zweischichtigen Verbundes als HochdruckMesselement. Der Ansatz der Energieerhaltung im Mehrlagenkörper ähnelt bereits vorgestellten Ansätzen 
[4, 5]. Das Bild 3 zeigt das Verbundelement mit den für den analytischen Ansatz wichtigen Variablen, wie den geometrischen Abmessungen und mechanischen Materialkonstanten.
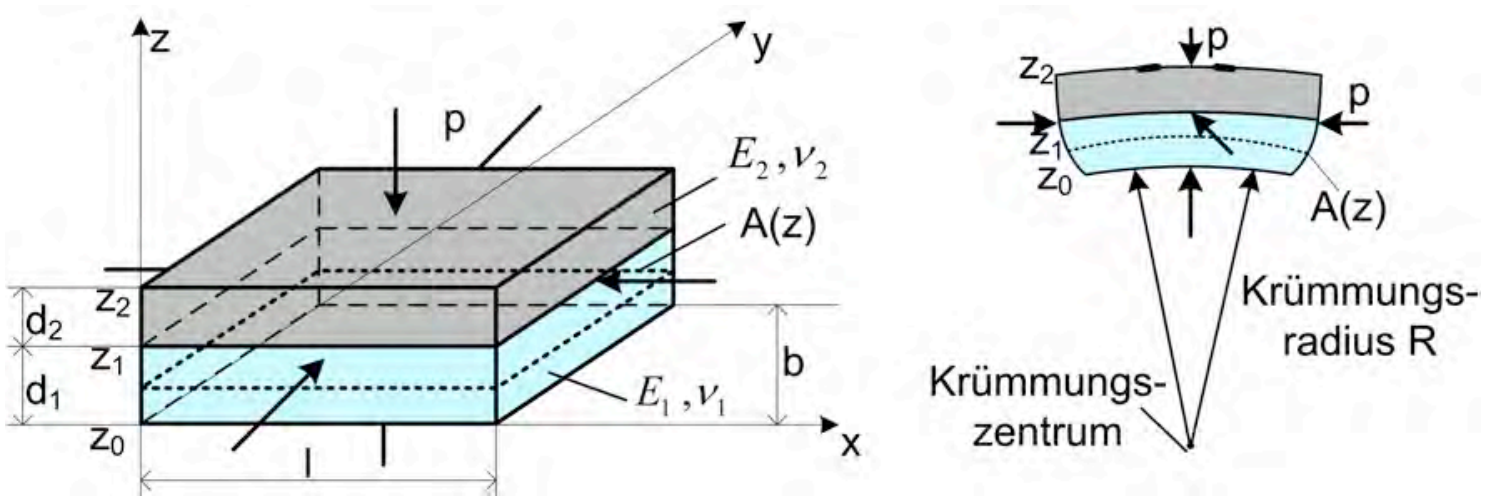

Bild 3: Verbundelement mit verwendeten Optimierungsparametern für den analytischen Ansatz im kartesischen Koordinatensystem: (rechts) infolge hydrostatischer Druckbelastung schalenförmig verbogenes Verbundelement mit eingezeichnetem Biegezentrum

Gleichung (1) zeigt den analytischen Ansatz zur Berechnung der gesamten im hydrostatisch belasteten Verbundelement gespeicherten Energie $W$. Diese ergibt sich durch Integration der in $z$-Richtung stetigen Dehnung $S$ und anschließender Summenbildung über den Energieinhalt der beiden Schichten:

$$
W=\sum_{i=1}^{n} \int_{z_{i-1}}^{\sigma_{1}}\left\{\frac{S^{2}(z) \cdot E_{i}}{2\left(1-2 v_{i}\right)}\right\} \mathrm{d} A \cdot \mathrm{d} z \quad \text {, mit dem Elastizitätsmodul } E \text { und der Querkontraktionszahl } v .
$$

Zur Lösung des Energieansatzes nach Gl. (1) wird angenommen, dass das Verbundelement aufgrund seiner allseitigen Druckbelastung ohne Einspannungsrandbedingungen betrachtet werden kann. Das Verbundelement ist demnach als mechanisch frei anzusehen und nimmt seine energetisch günstigste Lage im Raum ein. Dies ist das physikalische Minimum der potentiellen Energie. Die Minimalwertbetrachtung der im Verbundelement gespeicherten Energie liefert die Lösung des Ansatzes nach GI. (1). Die eigenen Veröffentlichungen $[2,3,6]$ liefern die Lösung für den Krümmungsradius $R$ und die Dehnung der Grundschicht $S_{z 0}$ für das allseitig belastete Verbundelement (Bild 3). Die Lösung des vorgestellten Ansatzes zeigt, dass der Messeffekt basierend auf der mechanischen Verspannung des Verbundelementes, hauptsächlich von folgenden Parametern abhängt: Position und Ausrichtung der Widerstände, Materialkombination (Elastizitätsmodul $E_{\mathrm{i}}$ und Querkontraktionszahl $v_{\mathrm{i}}$ ) und Dicken $d_{\mathrm{i}}$ der verbundenen Materialien.

\subsection{Schichtdickenoptimierung}

Die Materialkombination von Silizium als prozessiertem Chip und anodisch gebondetem Borosilikatglas als Substratgegenkörper und das Layout der Messelemente - Position und Lage der piezoresistiven Widerstände - bleiben zunächst unverändert. Mit der Lösung des analytischen Ansatzes ist es möglich, anhand des piezoresistiven Effekts und der berechneten Verteilung der mechanischen Spannung im Siliziumchip, die erwartete Empfindlichkeit eines Messelementes zu berechnen [7, 8, 9, 10]. Bild 4 zeigt diese errechnete normierte Empfindlichkeit, die für feste Siliziumdicken über einer sich ändernden Dicke des Glasgegenkörpers aufgetragen ist. Die Dicke des Silizium-Chips der vorhandenen Verbundelemente beträgt $d_{\mathrm{Si}}=390 \mu \mathrm{m} \pm 10 \mu \mathrm{m}$ einschließlich der Herstellungs- und Messtoleranz. 


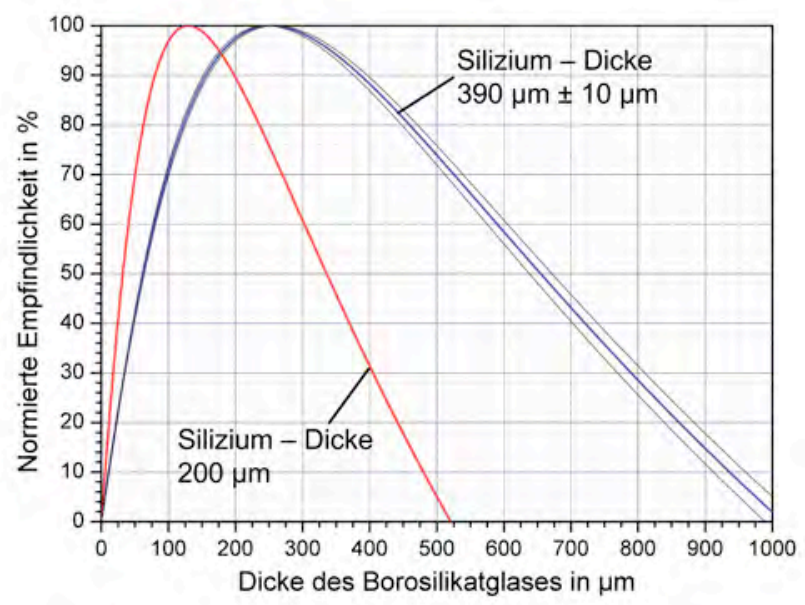

Bild 4: Anhand des analytischen Ansatzes berechnete normierte Empfindlichkeit des Verbundelementes für konstante Silizium-Dicken und variierende Dicke des Borosilikatglases als Substratgegenkörper

Die zweite dargestellte Kurve in Bild 4 zeigt die von der Gegenkörperdicke abhängige Empfindlichkeit für einen dünneren Silizium-Chip (Dicke $d_{\mathrm{Si}} \approx 200 \mu \mathrm{m}$ ). Es ist ebenso erkennbar, dass ein Maximum der Empfindlichkeit auftritt. Dieses physikalisch gegebene absolute Maximum lässt sich auf den angestrebten Zustand der minimalen potentiellen Energie zurückführen. So existiert ein bestimmtes, vom Verhältnis der mechanischen Konstanten der Materialparameter abhängiges Dickenverhältnis $D=\left(d_{\mathrm{Glas}} / d_{\mathrm{Si}}\right)$, bei welchem die Verbiegung des Verbundelementes maximal wird. Dieses Maximum der mechanischen Verspannung führt zu einer maximal möglichen Empfindlichkeit bei Variation des Parameters des Dickenverhältnisses. Dieser dargestellte Sachverhalt weist ein starkes Miniaturisierungspotential des Verbundelementes auf, ohne dabei an Empfindlichkeit einzubüßen.

Der Einfluss des Dickenverhältnisses von Silizium zu Substratgegenkörper ist ein entscheidendes Kriterium zur Einstellung der Empfindlichkeit des Messelementes. Anhand des in Bild 4 dargestellten Verlaufs liegt das Maximum der Empfindlichkeit für die vorhandenen Verbundelemente mit einer Silizium-Dicke von $d_{\mathrm{Si}}=390 \mu \mathrm{m} \pm 10 \mu \mathrm{m}$ bei ca. $d_{\mathrm{Glas}, \mathrm{opt}} \approx 250 \mu \mathrm{m} \pm 30 \mu \mathrm{m}$.

\section{$4 \quad$ Messtechnische Untersuchung von Verbundelementen mit verschiedenen Gegenkörperdicken im Hochdruckbereich bis $500 \mathrm{MPa}$}

\subsection{Fertigung von Messelementen mit verschiedenen Dickenverhältnissen}

Zur Herstellung der Verbundelemente kann das Borosilikatglas auf Waferebene in verschiedenen Standarddicken bezogen werden. Diese sind jedoch alle dicker, als das angegebene Optimum von $250 \mu \mathrm{m}$. Somit stellt ein nachträglicher Abdünnprozess eine Möglichkeit dar, die gewünschte Glasgegenkörperdicke einzustellen. Hierzu werden die jeweiligen Verbundelemente einzeln mit der prozessierten Oberseite des Silizium-Chips auf einen Trägerwafer aufgeklebt. Diese aufgeklebten Verbundelemente werden rückseitig einzeln in einem zeitaufwendigen Prozess durch mechanisches Abtragen der Glasschicht auf einer Läppmaschine bis zur vorgesehenen Glasdicke abgedünnt.

In [9] wurde anhand von Verbundelementen der 2. Generation mit einer ursprünglichen Glasdicke von $d_{\text {Glas,2.Gen }} \approx 480 \mu \mathrm{m} \pm 25 \mu \mathrm{m}$ und einem abgedünnten Messelement mit einer Glasdicke von ungefähr $250 \mu \mathrm{m} \pm 25 \mu \mathrm{m}$ die vorgestellte Schichtdickenoptimierung messtechnisch belegt. Die Abweichungen zwischen der erwarteten errechneten Steigerung der Empfindlichkeit und der gemessenen Empfindlichkeit war kleiner $1 \%$. 
Weitere Verbesserungen am Layout und somit der Position und Ausrichtung der piezoresistiven Widerstände im Verbundelement, führen zu Messelementen der 3. Generation mit deutlich gesteigerter Empfindlichkeit um ungefähr den Faktor 3,8 bei gleichem Schichtdickenverhältnis (Dicke des Glases ca. $480 \mu \mathrm{m})$ wie die vorgestellten Elemente der 2. Generation in [9].

Da Glas als Gegenkörpermaterial aufgrund seines amorphen Gefüges im Gegensatz zum äußerst stabilen kristallinen Aufbau von Silizium zum Fließen neigt, wird angenommen, dass es einen negativen Einfluss bei Linearität und Langzeitstabilität der Verbundelemente bewirkt. Da mit steigender Glasdicke und somit steigendem Dickenverhältnis $D$ das Glas einen höheren Einfluss auf das Verbundelement gewinnt, werden die Messelemente der 3. Generation an einen Glasgegenkörper mit einer Dicke von ca. $850 \mu \mathrm{m}$ gebondet, um den vermuteten Sachverhalt des starken Einflusses von Glas im Hochdruckbereich untersuchen zu können. Untersuchungen und somit Angaben zu den interessanten Alterungs- und Langzeitstabilitäts-Prozessen des Verbundelements können aufgrund der zu kurzen Zeit der Untersuchungen an dieser Stelle nicht präsentiert werden.

In dieser Veröffentlichung werden Messergebnisse an Verbundelementen der 3. Generation mit drei unterschiedlichen Dicken des Glasgegenkörpers präsentiert und verglichen. Die Tabelle 1 zeigt diese untersuchten Messelemente, die in drei unterschiedlichen Typen (A, B, C) je nach Dickenverhältnis eingeteilt sind. Zur Absicherung der Ergebnisse werden mehrere Messelemente je Typ aufgebaut. Für den unabgedünnten Typ A der 3. Generation mit ungefähr $850 \mu \mathrm{m}$ dickem Glasgegenkörper werden insgesamt drei Messelemente aufgebaut und verglichen. Die angegebenen Toleranzen für die Dicke des Silizium und des Gegenkörpers setzen sich zum einen aus unterschiedlichen Grunddicken des Ausgangsmaterials (Silizium-Wafer und Glasgegenkörper-Wafer) und Ungenauigkeiten bei der optischen Messung der Materialdicken auf das fertige Verbundelement zusammen.

Tabelle 1: Übersicht der untersuchten Messelement-Typen mit unterschiedlichem Dickenverhältnis

\begin{tabular}{lccc}
\hline Dicke von & Element Typ A & Element Typ B & Element Typ C \\
\hline Silizium Chip & $390 \mu \mathrm{m} \pm 3 \%$ & $390 \mu \mathrm{m} \pm 3 \%$ & $390 \mu \mathrm{m} \pm 3 \%$ \\
Glasgegenkörper & $850 \mu \mathrm{m} \pm 3 \%$ & $440 \mu \mathrm{m} \pm 5 \%$ & $310 \mu \mathrm{m} \pm 3 \%$ \\
Dickenverhältnis $D=\left(d_{\text {Glas }} / d_{\mathrm{Si}}\right)$ & 2,18 & 1,13 & 0,80 \\
Anzahl Messelemente & 3 & 3 & 1 \\
\hline
\end{tabular}

Die Elemente vom Typ B und C entstehen aus Verbundelementen des Typs A durch den beschriebenen Abdünnprozess auf einer Läppmaschine an einzeln aufgeklebten Verbundelementen. Während des Abdünnvorgangs kann die Gesamtdicke nur sehr ungenau bestimmt werden. Erst eine optische Dickenmessung im Anschluss an den Abdünnvorgang zeigt die erreichten Dickenverhältnisse, was zu größeren Toleranzbereichen der Gegenkörperdicken bei den abgedünnten Typen B und C führt. Während von Typ B wie auch von Typ A drei Messelemente untersucht werden, ist aufgrund von Defekten beim Abdünnvorgang nur ein Element des Typs $\mathrm{C}$ vorhanden.

\subsection{Messtechnische Untersuchung}

Die messtechnische Charakterisierung der vorgestellten sieben Messelemente vom Typ A, B und C wird am eigenen Hochdruck-Messplatz durchgeführt. Dieser ermöglicht eine statische Druckerzeugung bis 5.000 bar. Mittels eines vorhandenen Kolbenmanometers können diskrete Druckstufen in 500 bar Schritten mit einer Messunsicherheit von $5 \times 10^{-6}$ bezogen auf den maximalen Druckbereich von 5.000 bar erzeugt werden [11].

Es werden die stationären Kennlinien eines Messelementes aufgenommen, indem pro Nenndruckbereich Messungen mit mehreren Belastungsumläufen zwischen Atmosphärendruck und Nenndruck mit einer Zwischenstufe durchgeführt werden. So kann die Reproduzierbarkeit der Ergebnisse sichergestellt werden. Bild 5 zeigt stellvertretend eine Messkurve eines Umlaufes bis 5.000 bar an einem Verbundelement des Typs A. Die Vollbrücken der Messelemente werden mit einer konstanten Gleichspannung von 2,5 V gespeist. Die Messung findet bei Raumtemperatur von ca. $22{ }^{\circ} \mathrm{C} \pm 2{ }^{\circ} \mathrm{C}$ statt. 
Anhand der aufgenommenen Messdaten je Nenndruckstufe werden charakteristische Werte eines Messelementes, wie die normierte Empfindlichkeit und systematische Fehler, berechnet. Die normierte Empfindlichkeit $B_{0}$ berechnet sich nach Gleichung (2)

$$
B_{0}=\frac{u_{n}}{U_{0} \cdot p_{n}} \text { mit der Einheit } \frac{\mu \mathrm{V}}{\mathrm{V} \cdot \mathrm{bar}}
$$

mit dem Nennsignal $u_{\mathrm{n}}$, also die Brückenausgangsspannung bei Nenndruck, bezogen auf den Nenndruck $p_{\mathrm{n}}$ und die Speisespannung $U_{0}$. Die normierte Empfindlichkeit dient der besseren Vergleichbarkeit der Ergebnisse bei Messungen mit unterschiedlichem Nenndruck und Versorgung. Als systematische Fehler werden die reduzierten Fehler bezüglich der Linearität, der Hysterese und des Nullpunktes betrachtet.

Um Aussagen über den Einfluss von Glas im Hochdruckbereich zu erhalten, werden die Messungen an den Messelementen der verschieden Typen mit unterschiedlichen Glasdicken für schrittweise steigenden Nenndruck durchgeführt. Der Nenndruck wird in 1.000 bar Schritten von 1.000 bar bis 5.000 bar erhöht.

Tabelle 2 zeigt die charakteristischen Werte für Verbundelemente des Typs A mit dem dicksten Glasgegenkörper von $850 \mu \mathrm{m}$. Da insgesamt drei Messelemente des Typs A charakterisiert werden (Tabelle 1), sind in Tabelle 2 die Mittelwerte angegeben.

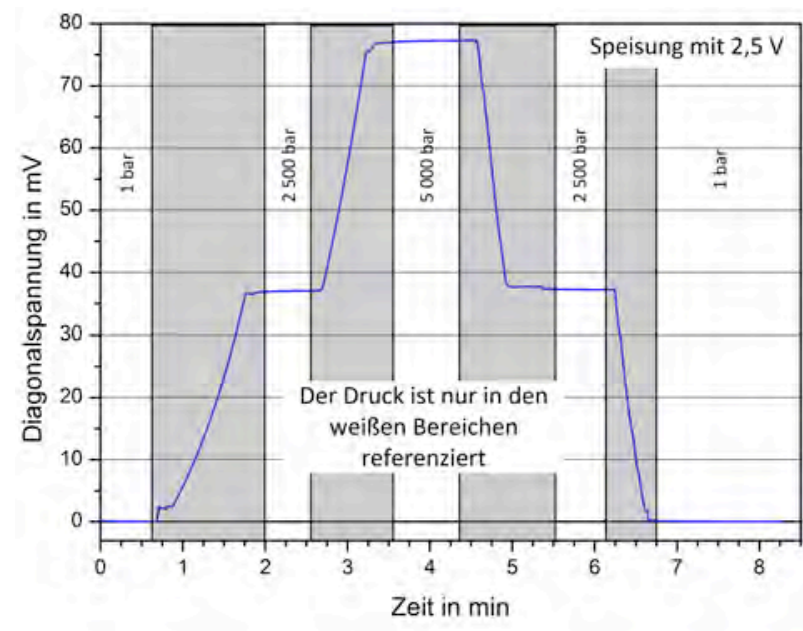

Bild 5: Typisches Messergebnis an einem Verbundelement des Typs A: 1 Umlauf mit Nenndruck 5.000 bar, Konstantspannungsspeisung von $2,5 \mathrm{~V}$ bei Raumtemperatur $22{ }^{\circ} \mathrm{C} \pm 2{ }^{\circ} \mathrm{C}$

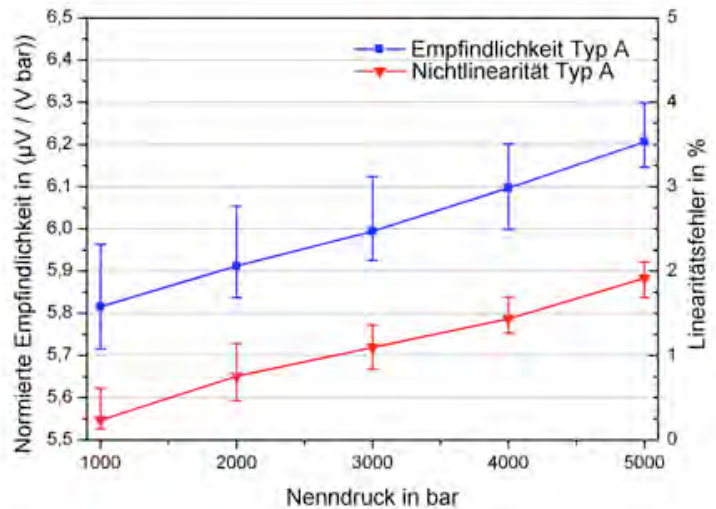

Bild 6: Normierte Empfindlichkeit und Linearitätsfehler von Verbundelementen des Typs A über steigendem Nenndruck; Mittelwert und Toleranzbereich

Da die Verbundelemente herstellungsbedingten Toleranzen aufweisen, streut die Empfindlichkeit um ca. $3 \%$. Im Bild 6 ist die normierte Empfindlichkeit wie auch der Linearitätsfehler der Verbundelemente des Typs A für steigenden Nenndruck inklusive der Toleranzbereiche dargestellt. Deutlich zu erkennen ist sowohl die Steigerung der Empfindlichkeit als auch die Zunahme des Linearitätsfehlers mit steigendem Nenndruck.

Tabelle 2: Charakteristische Werte des Elements Typ A bei Messung mit steigendem Nenndruckbereich

\begin{tabular}{lcccccc}
\hline Nenndruckbereich & bar & 1.000 & 2.000 & 3.000 & 4.000 & 5.000 \\
\hline Nennspannung & $\mathrm{mV}$ & 14,5 & 29,2 & 45,0 & 60,1 & 77,6 \\
Empfindlichkeit & $\mu \mathrm{V} / \mathrm{V}$ bar & 5,8 & 5,9 & 6,0 & 6,1 & 6,2 \\
Linearitätsfehler & $\%$ & 0,3 & 0,8 & 1,2 & 1,5 & 2,0 \\
Hysteresefehler & $\%$ & $-0,1$ & 0,1 & 0,1 & 0,1 & 0,1 \\
Nullpunktfehler & $\%$ & $-0,2$ & 0,2 & $-0,1$ & $-0,1$ & 0,1 \\
\hline
\end{tabular}


Die drei Messelemente des Typs B mit Glasgegenkörperdicken von ca. $410 \mu \mathrm{m}$ werden nach dem gleichen Schema bei steigendem Nenndruck charakterisiert. So zeigt Tabelle 3 die daraus abgeleiteten Kennwerte der Elemente Typ B. Auch für diesen Typ B sind die normierte Empfindlichkeit und der Linearitätsfehler über steigendem Nenndruck graphisch in Bild 7 dargestellt.

Anhand der Tabelle 3 und Bild 7 ist die erwartete Steigerung der absoluten Empfindlichkeit der Typ BElemente mit einem ausgewogenen Dickenverhältnis von ca. $D_{\text {Typ B }} \approx 1,1 \mathrm{im}$ Vergleich zu den Typ AElementen mit $D_{\text {Typ A }} \approx 2,1$ deutlich ersichtlich. Des Weiteren ist die Empfindlichkeit über dem steigenden Nenndruck betrachtet konstant, genauso wie die geringen Linearitätsfehler von maximal 0,26\% über dem steigenden Nenndruck hinweg.

Tabelle 3: Charakteristische Werte des Elements Typ B bei Messung mit steigendem Nenndruckbereich

\begin{tabular}{lcccccc}
\hline Nenndruckbereich & bar & 1.000 & 2.000 & 3.000 & 4.000 & 5.000 \\
\hline Nennspannung & $\mathrm{mV}$ & 32,5 & 64,1 & 97,9 & 130,5 & 162,7 \\
Empfindlichkeit & $\mu \mathrm{V} / \mathrm{V}$ bar & 12,7 & 12,8 & 12,8 & 12,8 & 12,7 \\
Linearität Fehler & $\%$ & 0,1 & 0,1 & 0,1 & 0,1 & 0,1 \\
Hysterese Fehler & $\%$ & 0,2 & 0,1 & 0,2 & 0,1 & 0,1 \\
Nullpunkt Fehler & $\%$ & 0,1 & $-0,2$ & $-0,2$ & $-0,1$ & 0,1 \\
\hline
\end{tabular}

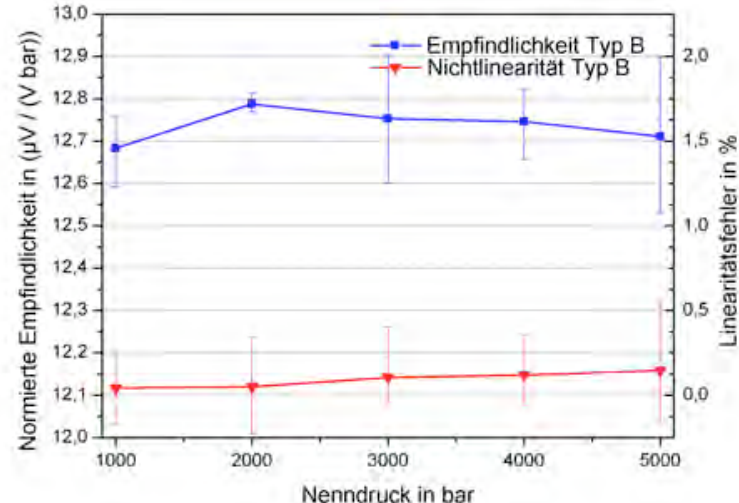

Bild 7: Normierte Empfindlichkeit und Linearitätsfehler von Verbundelementen des Typs B über steigendem Nenndruck; Mittelwert und Toleranzbereich

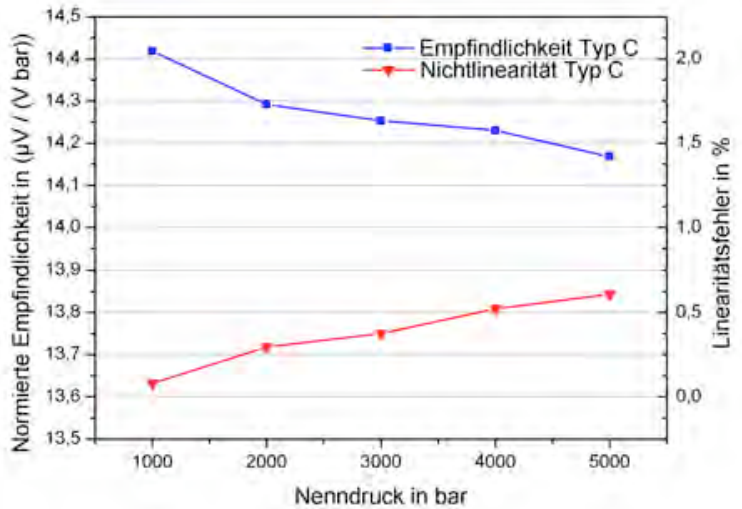

Bild 8: Normierte Empfindlichkeit und Linearitätsfehler von Verbundelementen des Typs C über steigendem Nenndruck

Zur messtechnischen Charakterisierung der Messelemente nach Typ C steht nur ein einzelnes Messelement zur Verfügung (Tabelle 1). Die anderen vorbereiteten Messelemente sind aufgrund von Defekten beim Abdünnvorgang, wie Ausbrüchen in Silizium bzw. Glas oder auch Risse im Messelement, nicht zu verwenden. Auch das charakterisierte Messelement des Typs $C$ hat kleine Defekte in Form von bis zu $30 \mu \mathrm{m}$ großen Ausbrüchen im Bereich des Glasgegenkörpers.

Da nur ein Messelement des Typs C zur Messung zur Verfügung steht, sind die in Tabelle 4 aufgezeigten Werte Mittelwerte über mehrere Messungen an diesem Messelement. Auf die Angabe von messelementabhängigen Toleranzbereichen wird in Bild 8 verzichtet. 
Tabelle 4: Charakteristische Werte des Elements Typ C bei Messung mit steigendem Nenndruckbereich

\begin{tabular}{lcrrrrr}
\hline Nenndruckbereich & bar & 1.000 & 2.000 & 3.000 & 4.000 & 5.000 \\
\hline Nennspannung & $\mathrm{mV}$ & 36,1 & 71,5 & 106,9 & 142,3 & 177,1 \\
Empfindlichkeit & $\mu \mathrm{V} / \mathrm{V}$ bar & 14,4 & 14,3 & 14,3 & 14,2 & 14,2 \\
Linearität Fehler & $\%$ & 0,1 & $-0,2$ & $-0,3$ & $-0,2$ & $-0,5$ \\
Hysterese Fehler & $\%$ & 0,0 & 0,1 & 0,1 & 0,3 & 0,2 \\
Nullpunkt Fehler & $\%$ & 0,3 & 0,0 & 0,0 & 0,0 & 0,0 \\
\hline
\end{tabular}

\subsection{Fazit der messtechnischen Untersuchung}

Die erwartete Steigerung der Empfindlichkeit anhand der Anpassung des Dickenverhältnisses durch rückseitiges Abdünnen des Glasgegenkörpers der Verbundelemente ist anhand der Messergebnisse deutlich nachvollziehbar. Um einen Vergleich der tatsächlichen Steigerung zur erwarteten Steigerung der Empfindlichkeit anhand des analytischen Ansatzes und des darstellenden Diagramms der Dickenabhängigkeit in Bild 4 durchführen zu können, werden die Messergebisse der Elemente Typ B mit mittlerer Glasdicke als Referenz genommen. Die Steigerung der Empfindlichkeit beim Vergleich der Elemente vom Typ B und dem Element vom Typ C, das dem berechneten Optimum des Dickenverhältnisses am nächsten liegt, entspricht unter Berücksichtigung der angegebenen Toleranzen der gemessenen Dicken dem erwarteten Wert.

Die Empfindlichkeit der Verbundelemente vom Typ A mit dem dicken Glasgegenkörper weichen jedoch weit von der erwarteten Kurve der Empfindlichkeit aus Bild 4 ab. Die Elemente sind um etwa den Faktor 2 empfindlicher als berechnet. Da der Glasgegenkörper mehr als doppelt so dick wie das Silizium-Chip ist (Dickenverhältnis der Verbundelemente vom Typ A: $D_{\text {Typ A }} \approx 2,1$, siehe Tabelle 1 ), wird vermutet, dass das weit von der Verbindungsschicht entfernt liegende Glas nur sehr geringe Wechselwirkungen mit dem Silizium eingeht. Die mechanische Verspannung des Verbundelementes als Messeffekt, breitet sich aufgrund der stetig verlaufenden Dehnung von der Verbindungsebene durch das Messelement aus. Umso weiter das Material von der Verbindungsebene entfernt liegt, umso geringeren Einfluss übt es auf die mechanische Verspannung und somit auf das mechanisch fehlangepasste andere Material des Verbundelementes aus. Daher funktioniert der analytische Ansatz zur Berechnung der Empfindlichkeit für Dickenverhältnisse von ungefähr 1 sehr gut. In dem Bereich des Dickenverhältnisses von 0,5 bis 1,5 sind die Abweichungen von der Berechnung ebenfalls gering, kleiner $2 \%$. Jedoch wird vermutet, dass die Kurve der Empfindlichkeit über der Glasdicke für eine feste Siliziumdicke, wie in Bild 4 dargestellt, ab ungefähr einem Dickenverhältnis von 1,5 beginnt abzuflachen und sich schließlich einem Endwert asymptotisch annähert. Der Nulldurchgang der Kurve der Empfindlichkeit wird für große Glasdicken, also für große Dickenverhältnisse, nicht erreicht. Der Nulldurchgang dieser Kurve auf der anderen Seite des Maximums, also für kleine Glasdicken / Dickenverhältnisse ist jedoch berechtigt. Besteht das Verbundelement im Grenzfall nur aus Silizium, so wird sich keine mechanische Verspannung, also ein Gradient der Spannung entlang des Messelementes einstellen. Die auftretende Kompression des Siliziums wird zu einer im gesamten Körper homogenen mechanischen Spannung führen. In Verbindung mit dem piezoresistiven Effekt würden sich für das bestehende technologische Layout alle Wiederstände der Vollbrücke gleichermaßen ändern. Eine Druckmessung mit den zur Vollbrücke verschalteten piezoresistiven Widerständen wäre nicht möglich, die Empfindlichkeit beträgt annähernd null.

Das Bild 9 zeigt für die unterschiedlichen untersuchten Messelement Typen A bis C mit entsprechenden Dickenverhältnissen nach Tabelle 1 die relative Änderung der Empfindlichkeit bezogen auf die Empfindlichkeit bei der Nenndruckstufe 1.000 bar. Die stabilste Empfindlichkeit über die untersuchten steigenden Nenndrücke bis 5.000 bar zeigen die Elemente vom Typ B mit dem mitteldicken Glasgegenkörper von ca. $440 \mu \mathrm{m}\left(D_{\text {Typ } \mathrm{B}} \approx 1,13\right)$ mit Schwankungen kleiner 1\%. Die Elemente vom Typ A mit dem dicken Glasgegenkörper $\left(D_{\text {Tyр A }} \approx 2,1\right)$ weisen eine kontinuierliche Steigerung der Empfindlichkeit bei steigendem Nenndruck bis zu ca. $7 \%$ bei 5.000 bar auf. Dies hängt eng mit der dargestellten Zunahme des Linearitätsfehlers über dem steigenden Nenndruck nach Bild 10 für die Typ A-Elemente zusammen. Absolut gesehen ist der Linearitätsfehler mit ungefähr $2 \%$ auch der größte auftretende in der Untersuchung für die Typ AElemente mit dem dicksten Glasgegenkörper bei dem höchsten Nenndruck. 


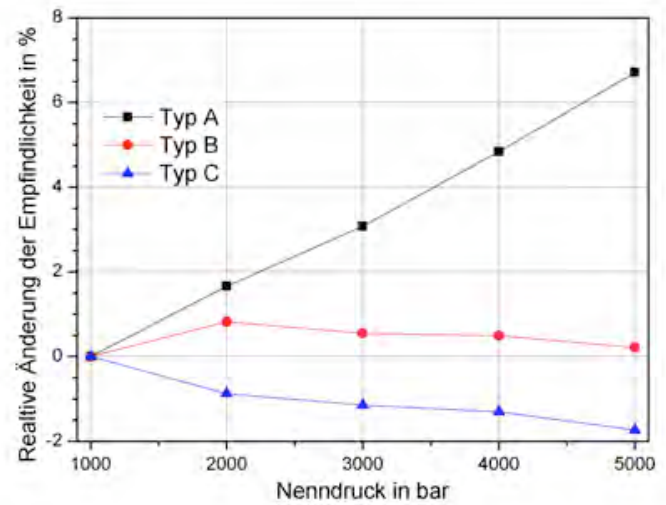

Bild 9: Relative Änderung der Empfindlichkeit bezogen auf die jeweilige Empfindlichkeit beim Nenndruck von 1.000 bar für die unterschiedlichen Messelement Typen A bis C

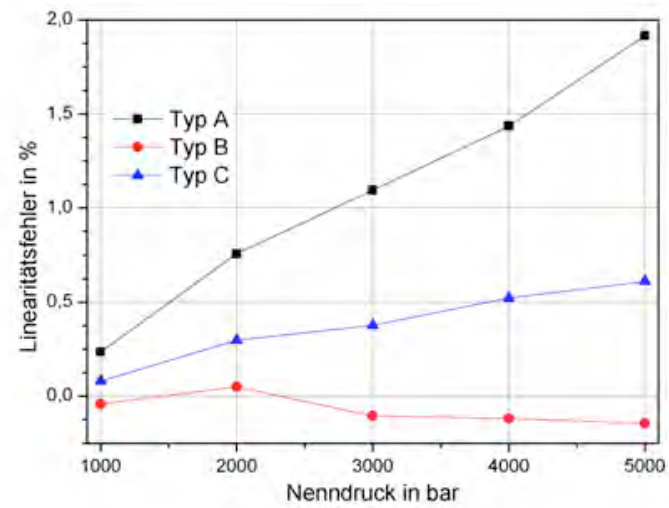

Bild 10: Linearitätsfehler für die unterschiedlichen Messelement-Typen A bis C

Die empfindlichsten Elemente vom Typ C mit dem vergleichsweise dünnsten Glasgegenkörper zeigen größere Abweichungen als die Typ-B Elemente. Die Empfindlichkeit nimmt mit steigendem Nenndruck um ca. $1,5 \%$ ab. Auch in diesem Fall spiegelt sich diese Änderung der Empfindlichkeit in der Darstellung des Linearitätsfehlers (Bild 10) wieder. Die dargestellten Ergebnisse des Typ C-Elements, das dem rechnerischen Optimum der Schichtdicken am nächsten liegt, sind mit Vorsicht zu bewerten, da nur ein Element verwendet werden konnte.

Zusammenfassend zeigen die Ergebnisse über den steigenden Nenndruck mit Elementen unterschiedlicher Dickenverhältnisse nach Bild 9 und 10 deutlich, dass die stärkste auftretende Nichtlinearität bei den Elementen mit dem dicksten Glasgegenkörper auftreten. Für die Typ A-Elemente mit einem Dickenverhältnis von $D_{\text {Tyр } A} \approx 2,1$ ist das Glas mehr als doppelt so dick wie das Silizium. Umso dicker der Glasgegenkörper gewählt wird, umso größeren Einfluss hat das Glas auf das Messelement. Somit zeigt sich der zunehmend nichtlineare Einfluss des Glases bezüglich der Kompression mit steigendem Druck im Hochdruckbereich immer deutlicher.

\section{$4 \quad$ Fazit und Ausblick}

In dieser Veröffentlichung wurde der messtechnische Nachweis des Einflusses der Dickenverhältnisse, die mathematisch beschrieben werden können, gebracht. Des Weiteren wurde durch die Untersuchung von Messelementen mit sehr dicken Glasgegenkörpern eine durch das Material Glas bedingte Nichtlinearität des Verhaltens von Glas im Hochdruckbereich gezeigt. Da das einzige untersuchte Verbundelement vom Typ C dicker als das angestrebte Optimum ist, konnte die maximal erwartete Empfindlichkeit nicht erreicht werden. Zudem war das Messelement durch den Abdünnprozess beschädigt.

Das vorgestellte Verbundelement als Hochdruck-Messelement bietet ein großes Potential zur weiteren Steigerung der Empfindlichkeit durch geplante Optimierungen am Layout und dem gezielten Einsatz des vielversprechendsten Dickenverhältnisses. Die somit erreichbaren Empfindlichkeiten lassen den Einsatz der Messelemente sogar im Normaldruckbereich mit einem Nenndruck von 100 bar zu. Die Empfindlichkeit wird dann deutlich geringer als vorgestellt sein, und in einem Bereich von Sensoren nach Stand der Technik mit metallischen DMS liegen, dafür bleibt die hohe Überlastfestigkeit von größer 5.000 bar unverändert. Die Überlastfestigkeit und die Empfindlichkeit sind beim Verbundelement nicht voneinander abhängig, d.h. eine weitere Steigerung der Empfindlichkeit führt nicht zu Einbußen bei der Überlastfestigkeit. Dies stellt einen weiteren großen Unterschied zu Sensoren nach Stand der Technik anderer Herstellungstechnologien dar. 


\section{Danksagung}

Wir danken der Fa. TDK-EPC AG \& Co. KG für die finanzielle Unterstützung des Projektes und die Präparation der Messelemente.

\section{Literatur}

[1] S. Stavroulis, R. Werthschützky: Neuartiger überlastfester piezoresitiver Silizium-Hochdrucksensor. In: tm - Technisches Messen. Vol. 70, pp. 199-205, München, Oldenbourg Wissenschaftsverlag, 2003

[2] P. Heinickel, R. Werthschützky: Functionality of a novel overload resistant silicon high-pressure sensing element. In: IEEE Transducers 2009 Conference Proceeding. Denver, CO, USA 2009.

[3] P. Heinickel, R. Werthschützky: Novel Silicon High-Pressure Sensing Element. In: Sensor + Test Conference 2009. Proceedings Sensor, Volume I. Nürnberg, Germany 2009.

[4] R. G. Ballas: Piezoelectric Multilayer Beam Bending Actuators. Heidelberg, Springer-Verlag GmbH, 2007.

[5] Schroth, A.; Gerlach, G. (editor): Modelle für Balken und Platten in der Mikromechanik. In: Dresdner Beiträge zur Sensorik. Vol. 1, Dissertation, Institut für Festköperelektronik, TU Dresden, Dresden University Press, 1996

[6] P. Heinickel, R. Werthschützky: Piezoresistives Silizium-Messelement zur Messung im HochdruckBereich bei 5.000 bar. In: XXIV. Messtechnisches Symposium des Arbeitskreises der Hochschullehrer für Messtechnik e.V., Shaker Verlag, Aachen, 2011

[7] Bao, M.-H.; Middelhoek, S. (editor): Handbook of Sensors and Actuators. Vol. 8: Micro mechanical transducers: pressure sensors, accelerometers and gyroscopes. 1st. edition, Amsterdam, Elsevier, 2004

[8] Pfeifer, G.; Werthschützky, R.; Trumpold, H. (Hrsg.); Woschni, E.-G. (Hrsg.): Drucksensoren. 1. Auflage, Berlin, VEB Verlag Technik, 1989

[9] P. Heinickel, R. Werthschützky: Effect of the thickness-ratio of the piezoresistive composite element for measuring high-pressure. Proc. Eurosensors XXIV, 2010, Linz, Österreich

[10] P. Heinickel, R. Werthschützky: High-pressure measurements at $500 \mathrm{MPa}$ of the novel piezoresistive composite element show the influence of glass counter body. Proc. Eurosensors XXV, 2011, Athen, Griechenland.

[11] DH Instruments, Inc.: PG7000 Piston Gauges - Operation and Maintenance Manual. Phoenix, AZ, 1998

[12] W. Hummel und E. Altstadt, "Spezial glasdurchf uhrungen und mehr", in Sensor Report, 4-2007, S. 27-28, Verlag Rek \& Thomas Medien AG, St. Gallen, 2007 